\title{
Evaluación de la toxicidad aguda de un extracto alcohólico de hojas de epazote (Chenopodium ambrosioides)
}

\author{
Rubí Zavala, MVZ1, José Herrera, $\mathrm{MVZ}^{3}$ Ana Silvia Lara, $\mathrm{MVZ}^{2}$, Víctor Da- \\ niel L. Garzón-Cortés, Ph.D ${ }^{1^{*}}$ \\ Instituto de Investigaciones Biomédicas, Universidad Nacional Autónoma de México (UNAM) Ciudad de México, \\ México \\ ${ }_{2}$ Facultad de Medicina Veterinaria y Zootecnia, Universidad Autónoma de Nuevo León (UANL), Nuevo León, \\ México \\ ${ }_{3}$ Centro de Investigaciones y Desarrollo en Ciencias de la Salud, Universidad Autónoma de Nuevo León (UANL) \\ Nuevo León, México
}

Recibido: 3 de noviembre del $2015 \quad$ Aprobado: 5 de marzo del 2016

*Autor de correspondencia: Víctor Daniel L. Garzón-Cortes. Instituto de Investigaciones Biomédicas, unam, tercer circuito exterior s/n, Coyoacán, Ciudad Universitaria, 04510 Ciudad de México, México. Teléfono: (+52 55) 562-28972. Correo electrónico: danielgarzon@biomedicas.unam.mx

Cómo citar este artículo: Zabala R, Herrera J, Lara AS, Garzón-Cortés VDL. Evaluación de la toxicidad aguda de un extracto alcohólico de hojas de epazote (Chenopodium ambrosioides). Spei Domus. 2016;12(24). doi: http://dx.doi.org/10.16925/ sp.v12i24.1890

Resumen. Introducción: la planta Chenopodium ambrosioides presenta una amplia distribución en América. En México, se conoce como "epazote", en la medicina alternativa su aceite es utilizado como un antihelmíntico, tanto para uso humano como animal. El objetivo de este trabajo fue evaluar el efecto del extracto de Chenopodium ambrosioides en una dosis de $2000 \mathrm{mg} / \mathrm{Kg}$ en ratas Long Evans para observar o descartar signos de toxicidad aguda o crónica. Metodología: se utilizaron diez ratas hembras Long Evans de 28 días de edad, divididas en dos grupos (control y experimental). Al grupo experimental se le administró el extracto por vía oral mediante una sonda orogástrica, con previo ayuno de 16 a 18 horas. Al grupo testigo se le administró solución salina. Resultados: el resultado de la administración del extracto no produjo mortalidad; aunque durante las primeras horas posadministración las ratas mostraron signos de letargo y somnolencia atribuibles a una leve toxicidad. En las observaciones posteriores, mostraron comportamientos similares a los del grupo control. Se observó un incrementó en cuanto al peso de los animales experimentales en ambos grupos Conclusiones: el extracto de la planta Chenopodium ambrosioides no posee toxicidad significativa administrada a la dosis utilizada (que marca el procedimiento de dosis fijas).

Palabras clave: animales de laboratorio, epazote, terapéutica, toxicidad. 


\title{
Acute toxicity evaluation of an alcoholic extract of epazote (Chenopodium ambrosioides) leaves
}

\begin{abstract}
Introduction: The plant Chenopodium ambrosioides is widely distributed in America. In Mexico, it is known as "epazote," in alternative medicine its oil is used as an anthelmintic, both for human and animal use. The objective of this work was to evaluate the effect of Chenopodium ambrosioides extract at a dose of $2000 \mathrm{mg} / \mathrm{kg}$ in Long Evans rats to observe or rule out signs of acute or chronic toxicity. Method: Ten 28-day-old Long Evans female rats were divided into two groups (control and experimental). The experimental group was administered the extract orally by an orogastric tube, after a fasting of 16 to 18 hours. Saline was given to the control group. Results: Administration of the extract did not cause mortality even though the rats showed signs of lethargy and drowsiness attributable to mild toxicity during the first post-administration hours. In subsequent observations, they showed behaviors similar to those of the control group. An increase in weight of experimental animals was observed in both groups. Conclusions: Chenopodium ambrosioides extract does not exhibit significant toxicity at the dose used (which marks the fixed dose procedure).
\end{abstract}

Keywords: laboratory animals, epazote, therapeutics, toxicity.

\section{Avaliação da toxicidade aguda de um extrato alcoólico de folhas de mastruz. (Chenopodium ambrosioides)}

Resumo. Introdução: a planta Chenopodium ambrosioides apresenta uma ampla distribuição na América. No México, é conhecida como "epazote", na medicina alternativa seu óleo é utilizado como um anti-helmíntico tanto para uso humano quanto animal. $\mathrm{O}$ objetivo deste trabalho foi avaliar o efeito do extrato de Chenopodium ambrosioides numa dose de $2000 \mathrm{mg} / \mathrm{Kg}$ em ratos Long Evans para observar ou descartar sinais de toxicidade aguda ou crônica. Metodologia: foram utilizados dez ratos fêmeas Long Evans de 28 dias de idade, divididas em dois grupos (controle e experimental). Ao grupo experimental, foi administrado o extrato por via oral mediante uma sondagem orogástrica, com jejum prévio de 16 a 18 horas. Ao grupo experimental, foi administrada solução salina. Resultados: o resultado da administração do extrato não produziu mortalidade; embora, durante as primeiras horas pós-administração, os ratos tenham mostrado sinais de letargia e sonolência atribuídos a uma leve toxicidade. Nas observações posteriores, mostraram comportamentos similares aos do grupo controle. Foi observado um aumento quanto ao peso dos animais experimentais em ambos os grupos. Conclusões: o extrato da planta Chenopodium ambrosioides não possui toxicidade significativa administrada à dose utilizada (que marca o procedimento de doses fixas).

Palavras-chave: animais de laboratório, mastruz, terapêutica, toxicidade. 


\section{Introducción}

La existencia y empleo de plantas con un elevado potencial terapéutico constituye una alternativa farmacológica de marcado interés debido a que podría favorecer el tratamiento de diversas patologías, de ahí la importancia de realizar estudios preclínicos para detectar posibles efectos tóxicos post administración [1]. En la actualidad existe escasa información acerca de la toxicidad de las plantas usadas en la medicina alternativa y/o tradicional. Tal es el caso de Chenopodium ambrosioides (epazote), la cual se distribuye ampliamente en México. Comúnmente llamada "epazote" o "paico" en países sudamericanos, esta es una planta vivaz aromática con diferentes usos. Sus hojas, raíces e inflorescencias son utilizadas como condimento o como planta medicinal, antihelmíntico, vermífugo, emenagogo y abortifaciente [2]. Otros de sus usos están señalados en la tabla 1 [3]. Originaria de América (usada más en Latinoamérica), donde los aztecas le dieron el nombre náhuatl de epazotl, epotl (hierba fétida) y tzotl (dulce), de donde proviene el nombre actual utilizado en México.

El epazote es una planta aromática perenne, más o menos pubescente, con el tallo usualmente postrado. Crece en suelos húmedos y bajos, de olor fuerte, con alrededor de cuarenta $\mathrm{cm}$ de altura. Sus hojas son oblongo-lanceoladas y serradas, de entre cuatro $\mathrm{cm}$ de longitud y uno $\mathrm{cm}$ de ancho, con pequeñas flores verdes en panículos terminales densos, cada uno con cinco sépalos. El cáliz persistente circunda al fruto y las semillas son negras y no mayores que $0,8 \mathrm{~mm}$ de longitud [4]. Su aceite esencial era uno de los antihelmínticos más utilizados tanto en humanos como en algunos animales domésticos, pero fue sustituido en la década de los años 40 por antihelmínticos menos tóxicos [5]. El aceite de esta planta es un líquido incoloro o amarillento de consistencia no muy viscosa, con un sabor amargo y un olor muy fuerte.

Se ha comprobado que es irritante a las mucosas gastrointestinales y una sobredosis causa efectos fatales sobre humanos y ratones [6], pero su efecto antihelmíntico era tan efectivo que a partir de ella, se desarrolló una importante industria alrededor del llamado "Aceite de Baltimore" [7].

Chenopodium ambrosioides ha demostrado efectividad antihelmíntica y antiprotozooaria sobre una gran cantidad de especies parasitarias tales como: Tripanosoma cruzi [8], Plasmodium [9]
Leishmania amazonensis [10], Ancylostoma duodenale, Trichuris trichuria y Ascarislumbricoides [11, 12]. En estudios sobre ganado caprino se comprobó la efectividad de C. ambrosioides sobre nemátodos del tracto gastrointestinal, entre ellos Haemonchus contortus. El tratamiento a corto plazo con el aceite esencial de la planta resultó ser inefectivo in vivo, pero in vitro reducía la viabilidad de los huevos, por lo que se planteó como una estrategia ecológica útil para reducir los niveles parasitarios en granjas [13]. Por otra parte, el tratamiento con Chenopodium ambrosioides ha sido efectivo al inhibir el crecimiento de tumores ascíticos en ratones [14]. Su principio activo (infusiones y derivados) es el ascaridol, su efecto tóxico radica en el cloruro de metileno, que presenta la mayor actividad relacionada al daño [15]. También se demostró que de las dos fracciones estudiadas (acuosas y cloruro metilénica), los principios activos responsables de la actividad citotóxica radican en la fracción orgánica, de la cual forma parte el ascaridol. Este último se encuentra presente en el aceite esencial en un $60-80 \%$ y en fresco, alrededor del $1 \%$, acompañado de niveles significativos de a-terpineno, su precursor [16]. Se realizó un estudio en el que se extraía el aceite esencial a distintos tiempos de maduración de la planta, demostrando que en los extractos jóvenes existía una mayor concentración de a-terpinenos y menor de ascaridol, mientras que en los extractos maduros la concentración de ascaridol era mayor a la de a-terpinenos [17].

En diciembre del 2002, la OECD (Organization for Economic Co-operation and Development) abolió la ejecución del test clásico para la determinación de toxicidad aguda y la dosis letal 50 (DL50) que fueron sustituidas por tres métodos alternativos cuyo objetivo común es reducir el número de animales empleados y minimizar el sufrimiento de los mismos. Estos métodos comprenden el test de Clasificación tóxica aguda o Método de las clases de toxicidad (Toxic Class Method, TCM), Procedimiento de sube y baja (Up-and-Down Method, UDP) y Procedimiento de dosis fijas [18].

El procedimiento de dosis fijas fue adoptado en la guía N. ${ }^{\circ} 420$ de la OECD como alternativa a la prueba clásica aguda. El método se basa en la evaluación de dosis prefijadas $(5,50,300$ y $2000 \mathrm{mg} / \mathrm{Kg})$ para discernir la clasificación tóxica de una sustancia según el Sistema Global Armonizado (sGa). La dosis seleccionada debe ser no letal, no dolorosa y 
no estresante, además de no ser corrosiva ni severamente irritante.

Los datos obtenidos por Medeiros et al [19] revelan que la infusión de hojas frescas de $C$. ambrosioides no producen toxicidad en ratas adultas hembras, con un tratamiento durante treinta días a dosis de $1000 \mathrm{mg} / \mathrm{Kg} /$ día.

El objetivo de este trabajo es descartar el efecto tóxico de la dosis máxima prefijada $(2000 \mathrm{mg} / \mathrm{kg})$ de un extracto de Chenopodium ambrosioides en ratas (Long Evans), así como revisar cualquier daño interno posterior a su administración.

Tabla 1. Usos recopilados para C. ambrosioides

\begin{tabular}{|l|l|}
\hline \multicolumn{1}{|c|}{ Uso } & \multicolumn{1}{c|}{ Localidad } \\
\hline Amebicida & Trinidad \\
\hline Analgésico & China \\
\hline Anemia & Colombia \\
\hline Artritis & China \\
\hline Asma & República Dominicana, \\
\hline & Panamá, Trinidad y Turquía \\
\hline Mordeduras & \\
\hline de insectos & China \\
\hline Disentería & Panamá y Trinidad \\
\hline Fungicida & Trinidad \\
\hline Narcótico & Estados Unidos \\
\hline Nervios & México, Turquía y Estados Unidos \\
\hline Estimulante & Trinidad y Turquía \\
\hline Cólicos & $\begin{array}{l}\text { Brasil, Chile, China, República Dominicana, } \\
\text { Tuatemala, Haití, México, Panamá, España, } \\
\text { zuela }\end{array}$ \\
\hline
\end{tabular}

Fuente: [3]

\section{Materiales y métodos}

\section{Obtención del extracto de Chenopodium ambrosioides}

El extracto etanólico se obtuvo a partir de una muestra de la planta medicinal C. ambrosioides. Se procede a pulverizar las hojas secas en una licuadora convencional, las cuales pasan por un filtro para eliminar los restos más grandes y se trabaja con residuo fino, del cual se toman 20 gr, (los cuales se colocaron en un matraz redondo), posteriormente se añaden $200 \mathrm{~mL}$ de etanol al $60 \%$, dejándolo en reposo durante siete días. Después de los siete días el extracto se seca en una estufa de convección a $70^{\circ} \mathrm{C}$. Con esto se obtiene el extracto seco de la muestra, el cual se lleva a una concentración de $200 \mathrm{mg} / \mathrm{ml}$ con agua destilada.

\section{Modelo biológico}

$\mathrm{Al}$ inicio del experimento se envió un protocolo detallado al Cicual (Comité Institucional de Cuidado de Animales de Laboratorio) del cidcs (Centro de Investigación y Desarrollo en Ciencias de la Salud) de la UANL. Este se estudió y se dio autorización para el inicio del protocolo. Posteriormente se compraron diez ratas hembra (raza Long Evans) adquiridas en el bioterio del Departamento de Inmunología (Facultad de Ciencias Biológicas, UANL) de 70 a $80 \mathrm{~g}$ al inicio del experimento y de 3 a 4 semanas de edad. Los animales se mantuvieron en un cuarto a una temperatura de $22 \pm 2{ }^{\circ} \mathrm{C}$ y ciclos de luz/oscuridad de 12/12 horas. Se les suministró alimento sólido comercial Marca LabDiet ${ }^{\circledR} 5013$ y agua apta para consumo humano, ambas ad líbitum. Los modelos biológicos fueron sometidos a observación previa en el área de cuarentena y posteriormente aclimatación durante siete días antes del experimento. Todos los procedimientos fueron realizados de acuerdo con la Norma Oficial Mexicana NOM-062-Zoo-1999 [20]. Y acordes con las 3R's de Rusell [21].

Los animales fueron identificados mediante la realización de marcas de tinta alrededor de la base de la cola en forma de anillo. El marcaje se realizó previo a la administración de la sustancia.

\section{Alojamiento}

Los animales se mantuvieron en jaulas de Makrolon 265X (150/180) x 420, con cama de aserrín. Se separaron en dos jaulas de a cinco para cada grupo. La distribución del espacio se decidió teniendo en cuenta edad, sexo y duración del experimento. La identificación de cada jaula se hizo por medio de tarjetas de papel en las cuales se indicaban los grupos TSCG y TSEg. Para este estudio se contó con el apoyo en las instalaciones de la Facultad de Medicina Veterinaria y Zootecnia de la UANL. 
Los animales se sometieron a cuarentena $y$ manejo, el cuidado diario de los mismos fue conducido por los investigadores a cargo sin cambio de personal.

\section{Procedimiento}

Para la realización del estudio, se siguió el método de dosis fijas, de acuerdo con la guía 420 de la Organización para la Cooperación y el Desarrollo Económico (OCDE). Aleatoriamente se conformaron dos grupos, los cuales se mantuvieron en cuarentena y aclimatación durante una semana. La sustancia de ensayo se administró con un ayuno previo de 16 a 18 horas por vía oral mediante una sonda orogástrica, a dosis de $2000 \mathrm{mg} / \mathrm{Kg}$. Se dosificaron las cinco hembras del grupo experimental, administrándoles un volumen de $10 \mathrm{~mL} / \mathrm{Kg}$ de masa corporal de la sustancia de ensayo. El grupo control recibió $0,5 \mathrm{~mL}$ de solución salina.

\section{Observaciones}

Se realizaron anotaciones sobre las observaciones de comportamiento de ambos grupos, a los treinta minutos y cuatro horas posteriores a la aplicación durante el primer día. Se continuó dando un seguimiento diario hasta catorce días después de la administración para determinar si la sustancia podía presentar un efecto a largo plazo. Se utilizó como referencia para la determinación del punto final los signos y tiempos de ocurrencia de los mismos, su comienzo y duración, considerando cambios en la piel, coloración de las membranas de mucosas y ojos, bradipnea, taquipnea, depresión o hiperactividad. Se prestó especial atención a la potencial aparición de temblores, convulsiones, salivación, diarrea, letargo, somnolencia y coma, así como a cualquier signo indicador de dolor en estos animales (movimiento y posición de bigotes, orejas, pelaje). Se registraron los pesos de los animales vivos los días 1, 4 y 14 del experimento, como parámetro demostrativo de toxicidad, entre otros.

Al concluir el experimento, se sacrificaron dos animales del grupo experimental y uno del grupo control por medio de sobredosis con pentobarbital. Se hizo una observación macroscópica de cerebro, corazón, bazo, hígado, pulmones y riñones. Se compararon los órganos del grupo control con los del tratamiento y se registraron las observaciones. No se aprecian cambios relevantes entre ambos grupos.

\section{Resultados}

El conjunto de observaciones realizadas durante el lapso del experimento, así como de la masa corporal como indicador de toxicidad, se registraron en tablas de datos para la realización de una curva y la comparación entre los grupos. La comparación de los pesos promedio del grupo tratamiento y del control se realizó con DMS (ANOVA).

\section{Toxicidad}

$\mathrm{Al}$ administrar el extracto a una dosis límite de 2000 $\mathrm{mg} / \mathrm{Kg}$ de masa corporal, la administración de la sustancia experimental no produjo mortalidad inmediata, aunque en las primeras horas hubo muestras de signos de letargo y somnolencia atribuibles a una leve toxicidad de acuerdo con la literatura especializada. Los estudios anatomopatológicos no revelaron alteración alguna en los órganos estudiados.

Los cambios de comportamiento observados no presentaron variaciones más que en las primeras horas en el grupo de tratamiento, comparado con el grupo control. Para el segundo día y durante el lapso del experimento, los animales continuaron con sus hábitos normales sin que ninguno de ellos presentara cambios o alteraciones.

Las observaciones realizadas (para los días establecidos) mostraron signos clínicos de letargo, sin llegar a presentar un dolor evidente o un comportamiento anormal después de cuatro horas posteriores a su administración (tabla 2).

Tabla 2. Signos clínicos observados en el grupo experimental después de la administración del extracto de Chenpodium ambrosioides

\begin{tabular}{|l|l|}
\hline \multicolumn{1}{|c|}{$\begin{array}{c}\text { Observación } \\
\text { (Tiempo) }\end{array}$} & \multicolumn{1}{c|}{ Signos clínicos } \\
\hline $\begin{array}{l}\text { Media hora post admi- } \\
\text { nistración }\end{array}$ & Letargo y somnolencia \\
\hline $\begin{array}{l}\text { Tres horas post admi- } \\
\text { nistración }\end{array}$ & $\begin{array}{l}\text { Signos de recuperación, recupe- } \\
\text { ración de apetito }\end{array}$ \\
\hline $\begin{array}{l}\text { Cuatro horas post } \\
\text { administración }\end{array}$ & $\begin{array}{l}\text { Reflejos normales, comporta- } \\
\text { miento de aseo, apetito normal, } \\
\text { consumo de agua normal }\end{array}$ \\
\hline Segundo día & $\begin{array}{l}\text { Comportamiento normal, no } \\
\text { hay signos de somnolencia }\end{array}$ \\
\hline $\begin{array}{l}\text { Tercer día hasta el día } \\
\text { final del estudio }\end{array}$ & $\begin{array}{l}\text { No hay signos de toxicidad, } \\
\text { comportamiento de hábitos, } \\
\text { aseo y alimentación normal }\end{array}$ \\
\hline
\end{tabular}

Fuente: elaboración propia 
La Guía 420 de la OCDE establece que si el análisis macroscópico en los animales sobrevivientes después de las 24 horas de iniciado el estudio revela datos patológicos, debe procederse al análisis microscópico; en este caso, al no encontrar evidencias de alteración, se prescindió de dicho estudio.

\section{Peso corporal}

Los pesos registrados del grupo experimental los días 1,4 y 14 fueron los siguientes, consignados en las tablas 3 y 4 .

Tabla 3. Pesos obtenidos los días 1,4 y 14 del grupo TSCG

\begin{tabular}{|c|c|c|c|c|}
\hline TSCG & Día 1 & Día 4 & Día 14 & $\begin{array}{c}\text { Ganancia } \\
\text { total de peso } \\
(\mathrm{g})\end{array}$ \\
\hline 1 & 80 & 101 & 134 & 54 \\
\hline 2 & 60 & 82 & 116 & 56 \\
\hline 3 & 44 & 63 & 106 & 62 \\
\hline 4 & 64 & 82 & 111 & 47 \\
\hline 5 & 45 & 65 & 111 & 66 \\
\hline Promedio & 58,6 & 78,6 & 115,6 & 57 \\
\hline
\end{tabular}

Fuente: elaboración propia

Tabla 4. Pesos obtenidos los días 1, 4 y 14 del grupo TSEG

\begin{tabular}{|c|c|c|c|c|}
\hline TSEG & Día 1 & Día 4 & Día 14 & $\begin{array}{c}\text { Ganancia } \\
\text { total de peso } \\
\text { (g) }\end{array}$ \\
\hline 1 & 72 & 93 & 126 & 54 \\
\hline 2 & 70 & 87 & 113 & 43 \\
\hline 3 & 69 & 93 & 129 & 60 \\
\hline 4 & 80 & 98 & 129 & 49 \\
\hline 5 & 71 & 88 & 114 & 43 \\
\hline Promedio & 72,4 & 91,8 & 122,2 & 49,8 \\
\hline
\end{tabular}

Fuente: elaboración propia

Las ratas no mostraron un descenso de peso después de la administración. al contrario siguieron su aumento de forma regular, manteniendo y casi igualando el peso del grupo control al finalizar la toma de datos (figura 1).

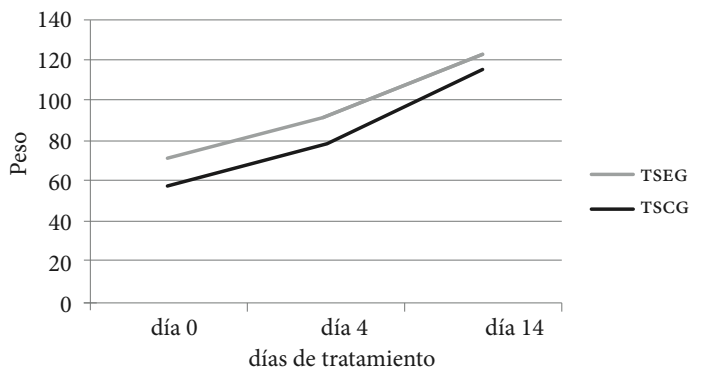

Figura 1. Comparación de pesos entre TSCG y TSEG Fuente: elaboración propia

\section{Discusión}

La administración del extracto alcohólico de las hojas de epazote confirmó la hipótesis planteada, al no presentarse signo alguno que indicara la toxicidad en los grupos de ratas a las cuales les fue administraba la dosis máxima.

Los resultados no arrojaron una diferencia significativa entre los grupos (figura 1) debido a que la concentración de componentes de la planta no fue suficiente para provocar un efecto que se reflejara en el promedio de ganancia diaria de peso de los animales, lo que ayuda a descartar la toxicidad de este extracto. Esto muestra resultados similares a los presentados por Medeiros et al. [19] al administrar una dosis de $1000 \mathrm{mg} / \mathrm{kg}$.

Los animales se mantuvieron bajo ciclos de luz y oscuridad controlados, diariamente se les proporcionó manejo para remarcar los anillos en la base de la cola, lo cual contribuyó a que los animales se mantuvieran tranquilos y sin signos de estrés. Con ello se descartaron problemas o factores externos atribuibles a los modelos biológicos que pudiesen interferir con los resultados.

Los datos obtenidos muestran una ausencia de toxicidad de la planta $C$. ambrosioides en base a los parámetros de peso y comportamiento analizados. Estos resultados ayudan a descartar posibles efectos secundarios derivados de su utilización en la medicina tradicional en humanos. Es importante aclarar que no se descartan variables en otras muestras de plantas obtenidas en localidades distintas a Nuevo León, México, ya que la planta de la cual se obtuvo el extracto fue adquirida de manera 
comercial en dicha ciudad y no se tiene un control sobre los componentes de este tipo de plantas utilizadas en toda Latinoamérica, debido a que la concentración de ascaridol varía dependiendo de la edad y la zona de obtención de la muestra [17] Se recomienda realizar estudios posteriores para corroborar la hipótesis.

\section{Conclusión}

Con base en los resultados obtenidos, los procedimientos mostrados en la Norma Oficial Mexicana NOM062-zoo-1999 y en el protocolo N. ${ }^{\circ} 420$ de la OCDE, se concluye que la sustancia activa ascaridol, extraída de una muestra de planta Chenpodium ambrosioides, en Monterrey Nuevo León, no posee toxicidad significativa administrada en una dosis máxima.

\section{Referencias}

[1] Bermúdez D, Monteagudo E, Boffill M, Díaz LE, Roca A, Betancourt E, Sileveira E. Evaluación de la toxicidad aguda de extractos de plantas medicinales por un método alternativo. REDVET. Revista electrónica de Veterinaria 1695-7504 [internet]. 2007 Marzo;8(3). Disponible en: http://www.veterinaria.org/ revistas/redvet/n030307/030706.pdf

[2] Curtin LSM. Healing Herbs of the Upper Rio Grande. Los Angeles, USA: Southwest Museum; 1965.

[3] Taylor L. The healing power of Rainforest herbs. Garden City Park, USA: Square One Publishers, Inc; 2005.

[4] Jaramillo E, Beatriz C, Duarte R, Edisson DW. Bioactividad del aceite esencial de Chenopodium ambrosioides colombiano. Rev Cubana Plant Med Ciudad de la Habana. 2012 ene-mar; 17(1): 54-64.

[5] Quinlan MB, Quinlan RJ, Nolan JM. Ethnophysiology and herbal treatments of intestinal worms in Dominica, West Indies. Journal of Ethnopharmacology. 2002;80(1):75-83.

[6] De Pascual TJ, Torres BC, Pérez MA. Essential oil of Chenopodium ambrosioides. Riv. Ital. Ess. 1980;62(1):123-125.

[7] Macdonald D, Vancrey K, Harrison P, Rangachari PK, Rosenfeld, Warren C, Sorger G. Ascaridole-less infusions of Chenopodium ambrosioides contain a nematocide(s) that is (are) no toxic to mammalian smooth muscle. J. Ethnopharmacol. 2004;92(23):215-221.

[8] KiuchiF, Itano Y, Uchiyama N, Honda G, Tsubouchi A, Nakajima-shimada J, Aoki T. Monoterpene hydrop- eroxides with trypanocidal activity from Chenopodium ambrosioides. J. Nat. Prod., 2002;65(4):509-512.

[9] Pollack Y, Segal R, Golenser J. The effect of ascaridole on the in vitro development of Plasmodium falciparum. Parasitol. Res. 1990;6(7):570-572.

[10] Patricio FJ, Costa GC, Pereira PVS, Aragão-Filho WC, Sousa SM, Frazão JB, et al. Efficacy of the intralesional treatment with Chenopodium ambrosioides in the murine infection by Leishmania amazonensis. J. Ethnopharmacol. 2008; 115(2):313319. doi:10.1016/j.jep.2007.10.009

[11] Giove NRA. Traditional medicine in the Treatment of enteroparasitosis. Rev. astroenterol. 1996;16(1):197202.

[12] Franca F, Lago EL, Marsden PD. Plants used in the treatment of leishmanial ulcers due to Leishmania (Viannia) braziliensis in an endemic area of Bahia. Rev. Soc. Bras. Med. Trop. 1996;29(3):229-232.

[13] Ketzis JK, Taylor A, Bowmanb DD, Browna DL, Warnickc LD, Erbc HN. Ambrosioides and its essential oil as treatments for Haemonchus contortus and mixed adult-nematode infections in goats. Small Rumin. Res. 2002;44(3):193-200. doi:10.1016/ S0921-4488(02)00047-0

[14] Nascimento FRF, Cruz GVB, Pereira PVS, Maciel MCG, Silva LA, Azaevedo APS, et al. Ascitic and solid Ehrlich tumor inhibition by Chenopodium ambrosioides L. treatment. Life Sciences. 2006;(78):26502653.

[15] Gadano A, Gurni A, López P, Ferraro G, Carballo M. In vitro genotoxic evaluation of the medicinal plant Chenopodium ambrosioides L. J. Ethnopharmacol. 2002;81(1):11-16.

[16] Gómez JR. Epazote (chenopodium ambrosoides). Revisión de sus características morfológicas, actividad farmacológica y biogénesis de su principal principio activo, ascaridol. Boletín Latinoamericano y del Caribe de Plantas Medicinales y Aromáticas. 2008;7(1): 3-9.

[17] Johnson MA, Croteau R. Biosynthesis of ascaridole: Iodide peroxidase-catalyzed synthesis of a monoterpene endoperoxide in soluble extracts of Chenopodium ambrosioides fruit. Arch. Biochem. Biophys. 1984; 235(1):254-266.

[18] OCDE, Organización para la Cooperación y el Desarrollo Económico. OECD Guideline for Testing of Chemicals. Acute Oral Toxicity - Fixed Dose Procedure. Guideline 420. 2001 dic 17. Disponible en: https:/ntp.niehs.nih.gov/iccvam/suppdocs/feddocs/ oecd/oecd_g1420.pdf

[19] Ucella de MI, Fonseca Figueiredo IM, Nunes OC, SchwarzAline. Toxicity of Chenopodium ambrosioides L. in rats: subchronic and reproductive studies. Biologia e Farmacia. 2011;06(02):152-160. 
[20] Secretaría de Agricultura, Ganadería, Desarrollo Rural, Pesca y Alimentación. Norma Oficial Mexicana NOM 062 - zoo - 1999. Especificaciones técnicas para la producción, cuidado y uso de los animales de laboratorio. México: Estados Unidos Mexicanos;
1999. Disponible en: http://www.ibt.unam.mx/computo/pdfs/bioterio.NOM-062.pdf

[21] Russell WMS, Burch RL. Principles of Humane Experimental Technique. Londres: Methuen; 1959. 